\title{
SCIDoC
}

\author{
International Journal of Dentistry and Oral Science (IJDOS) \\ ISSN: 2377-8075
}

\section{Interproximal Contact Area and Width: Relationship to Periodontal Parameters}

\section{Research Article}

Normah Yacob ${ }^{1}$, Raja Azman Awang ${ }^{2 *}$, Mohd Zulkarnain Sinor ${ }^{2}$

${ }^{1}$ Klinik Pergigian Jalan Gambut, Ministry of Heath, Malaysia.

${ }^{2}$ School of Dental Sciences, Health Campus, Universiti Sains Malaysia, 16150 Kubang Kerian, Kelantan, Malaysia.

\section{Abstract}

Objective: This study focuses on investigating the influence of the interproximal contact area and width on periodontal parameters.

Materials and Methods: A total of 30 periodontitis subjects were involved in the study, and 661 teeth were selected for analysis. Periodontal parameters such as plaque score, gingiva score, clinical probing depth and radiographic bone level were recorded for each tooth. Upper and lower impressions were taken for construction of study models which were used for the measurement of interproximal contact area and width. simple linear regression (SLR) and multiple linear regressions (MLR) analysis were used to evaluate the relationship between periodontal parameters and interproximal dimension.

Results: The SLR analyses consistently showed that the interproximal contact area and width were both significantly related with plaque score, gingiva score and bone level, and MLR analyses confirmed that both the interproximal contact area and width were significant predictors for each plaque score, gingiva score and bone level model.

Conclusion: Within the limitation of the study, we can conclude that the interproximal contact area and width have a significant relationship with the presence of dental biofilm, gingival bleeding and bone level in the subjects susceptible to periodontal disease.

Keywords: Periodontitis; Periodontal Disease; Gingivitis; Interdental Papilla; Periodontal Index; Dental Plaque Index; Periodontal Pocket.

\section{Introduction}

The periodontal disease initiation and progression is mainly influenced by the shifts and stability of dental biofilm community [1]. However, not all patients with equal exposure to dental biofilm community are susceptible to the disease. Furthermore, it has been shown that in patients who were susceptible to periodontitis, not all of their teeth were equally affected by the disease [2]. Studies have demonstrated that the retentiveness, maturity and composition of dental biofilm are highly variable in each colonised tooth, which eventually alters the periodontium health [3]. These variabilities were found to be associated with local factors such as oral hygiene, tooth malposition, tooth anatomy and gingival contours $[4,5]$.

It is perceived that periodontitis onset and progression occur more frequently at the interproximal surface [6]. The dimension of the interproximal area appears to play some roles in the establishment of the periodontal disease. This dimension is determined by an area of interproximal contact, a horizontal distance between neighbouring teeth and vertical distance from the alveolar bone crest [7], which is occupied by interdental papilla. The papilla is an essential biological barrier that protects the periodontal structure, and also acts as a physical barrier against food impaction. The imperfection in shape and size of the interproximal dimension may lead to problems in maintaining the integrity of the interproximal area, which could lead to dental biofilm and later on periodontal disease. However, to the best of our knowledge, there is limited study, if any, on the relationship between the interproximal dimension and periodontal variables.

Most of the clinical studies to date investigate the inter-subject

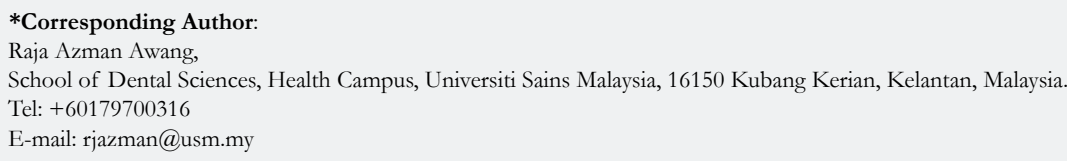

Copyright: Raja Azman Awang 2020 . This is an open-access article distributed under the terms of the Creative Commons Attribution License, which permits unrestricted use, distribution and reproduction in any medium, provided the original author and source are credited. 
relationship between dental biofilm retentive factors and periodontal health, where subjects are simply grouped into cohorts of health and disease. This is quite problematic as the individual heterogeneity is not considered in the analysis [8]. Therefore, intra-subject studies investigating periodontal health in the same patient are invaluable [9]. Unfortunately, studies that use this approach are lacking, and no studies to date have investigated the relationship between the plaque retentive factors and periodontal disease using this method.

In clinical settings, understanding the relationship between local factors such as interproximal dimension and periodontal disease is essential in communication between clinician and patient about oral health education and treatment plan. Personalised oral hygiene regimen can be formulated and proposed if it is proven that these local factors do have an impact on disease progression. It is generally agreed that periodontitis can be prevented, easily diagnosed and successfully controlled if appropriate prevention, diagnosis and treatment are applied [10]. Periodontal diagnosis should include a thorough assessment of possible risk factors to promote early detection of disease and providing the earliest treatment possible. This study focuses on investigating the influence of interproximal contact area and width on the periodontal parameters including plaque score, gingiva score, clinical probing depth and bone level.

\section{Materials and Methods}

A total of 17 men and 13 women were involved in the study, and 661 teeth were selected for analysis. The subjects were periodontitis patient with basic periodontal examination (BPE) score of at least 3 or 4 at one of the sextant and had not lost more than 4 teeth. All subjects did not receive periodontal therapy within the last six months and had no underlying medical problems. Written informed consent was obtained from all subjects after the study had been explained. The study was approved by the Human Research Ethics Committee (JEPeM) of Universiti Sains Malaysia (USM/JEPeM/16110483).

All teeth, except wisdom teeth, were subjected to evaluation of clinical and radiographic periodontal parameters, which include plaque score, gingiva score, clinical probing depth and radiographic bone level. An impression of the upper and lower jaw was then taken using alginate impression material (Kromopan, Lascod S.P.A. Lab., Italy). Dental models were constructed using type III dental stone, Pro - Solid ${ }^{\circledR}$ (Saint-Gobain Formula, Germany), which were later used for measurement of the interproximal con- tact area and width. Prior to the start of actual measurement, a calibration session was held to enumerate intra-examiner reliability for measurement of periodontal parameters. In all analyses, the percent agreement and kappa score were between 90 to $100 \%$ and 0.80 to 1.00 , respectively.

\section{Measurement of periodontal parameters}

The plaque score was assessed using O'Leary plaque index [11], and the gingiva score was assessed using Ainamo's Gingival Bleeding Index [12]. Four surfaces were evaluated for each tooth, which was mesial, distal, facial and lingual/palatal. The tooth plaque and gingival score were either $0,0.25,0.5,0.75$ or 1 , based on the number of a surface with plaque or gingival bleeding.

Clinical probing depth (CPD) was recorded at six sites of a tooth: mesiobuccal, distobuccal, mesiolingual, distolingual, midlingual and midbuccal, and the average CPD was the tooth's pocket depth score. Furthermore, the dental panoramic tomography (DPT) image of each subject was used for the measurement of a radiographic bone loss, which is a distance between the cementoenamel junction and bone crest, using the Image J software (National Institutes of Health, University of Wisconsin). The demarcation between a more radiodense enamel and less radiodense root cementum in the cervical region of teeth in the radiograph was marked as the cementoenamel junction [13].

\section{Measurement of interproximal dimension}

The interproximal contact area is the zone in which two adjacent teeth meet (Foulger et al., 2010). It was measured as a distance in millimetres between the apical point (AP) and the occlusal point (OP) of the contact area, using a digital calliper (Figure 1). The $\mathrm{AP}$ and $\mathrm{OP}$ were references for the interdental papilla tip and initiation of the interdental occlusal embrasure, respectively [14]. For the measurement of interproximal width, gingival zenith, the most apical part of gingiva scallop was marked as a reference point (RP). The interproximal width was measured as the horizontal distance between the RP of two adjacent teeth. The interproximal width values were assigned to the tooth on the mesial [15].

\section{Statistical analysis}

Statistical analyses were carried out using IBM SPSS (Statistical Package for the Social Sciences) Version 24.0. The influence of the interproximal contact area and width on periodontal param-

Figure 1. Measurement of interproximal area (AP - OP) and width (RP - RP). AP = apical point, $\mathrm{OP}=$ occlusal point, and $\mathbf{R P}=$ reference point.

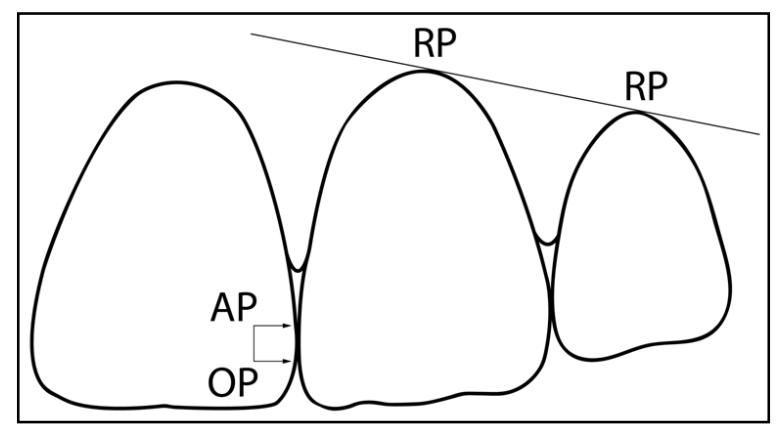


eters were analysed using simple linear regression (SLR) and stepwise multiple linear regressions (MLR) analysis. The unmeasurable surface on the dental model and radiograph was reported as a missing value. Significant level was set at $\mathrm{p}=0.05$.

\section{Results}

From 30 participants involved, 661 teeth were selected as study samples. The prevalence of periodontally affected teeth (CPD $\geq$ $4 \mathrm{~mm}$ ) in this study samples was $56.7 \%$, which was comparable to $43.3 \%$ prevalence of the healthy gingiva/gingivitis (CPD $<$ $4 \mathrm{~mm}$ ). The mean (standard deviation) plaque score and gingiva score were $0.49(0.35)$ and $0.48(0.34)$, respectively. Detail description of the study sample is shown in Table 1.

Simple (SLR) and multiple linear regression (MLR) analyses were carried out to investigate the relationship between the interproximal dimension and periodontal parameters (Table 2). The SLR analyses consistently showed that the interproximal contact area and width were both significantly related with plaque score, gingiva score and bone level, and MLR analyses confirmed that both the interproximal contact area and width were significant predictors for each plaque score, gingiva score and bone level model. Only in the clinical probing depth model, the interproximal contact area was found not a significant predictor.

Table 1. Description of study samples.

\begin{tabular}{|c|c|c|}
\hline Variables & Mean (SD) & Frequency (\%) \\
\hline $\begin{array}{c}\text { Intra-arch location of tooth } \\
\text { Anterior } \\
\text { Posterior }\end{array}$ & & $346(52.3)$ \\
Inter-arch location of tooth & & $315(47.7)$ \\
Maxilla & & $333(50.4)$ \\
Mandible & & $328(49.6)$ \\
\hline Clinical probing depth & & \\
CPD $\geq 4 \mathrm{~mm}$ & & $375(56.7)$ \\
CPD $<4 \mathrm{~mm}$ & & \\
\hline Periodontal variables & & \\
Plaque score & $0.49(0.35)$ & \\
Gingiva score & $0.48(0.34)$ & \\
CPD & $2.76(1.05)$ & \\
Bone level $^{\mathrm{b}}$ & $10.99(5.08)$ & \\
\hline Interproximal dimension $_{\text {Interproximal contact area }}^{\mathrm{a}}$ & $3.4(2.20)$ & \\
Interproximal width $^{\mathrm{a}}$ & $7.35(3.03)$ & \\
\hline
\end{tabular}

$\mathrm{n}=661 ;^{\mathrm{a}}=2$ missing values; ${ }^{\mathrm{b}}=132$ missing values; SD: standard deviation; CPD: clinical probing depth.

Table 2. Relationship between interproximal dimension and plaque score using simple and multiple linear regression.

\begin{tabular}{|c|c|c|c|c|c|c|}
\hline \multirow[b]{2}{*}{ Variables } & \multicolumn{3}{|c|}{ SLR } & \multicolumn{3}{|c|}{ MLR } \\
\hline & $\begin{array}{c}\text { B } \\
(95 \% \mathrm{CI})\end{array}$ & $\underset{\text { (t-stats) }}{\mathbf{R}^{2}}$ & $\begin{array}{c}p \\
\text { value }\end{array}$ & $\begin{array}{c}\text { Adj- } \beta \\
(95 \% \text { CI })\end{array}$ & t-stats & $\begin{array}{c}p \\
\text { value }\end{array}$ \\
\hline $\begin{array}{c}\text { Plaque score } \\
\text { IPA } \\
\text { IPW }\end{array}$ & $\begin{array}{c}-0.03(-0.04,-0.02) \\
0.02(0.01,0.02)\end{array}$ & $\begin{array}{c}0.03(-4.17) \\
0.02-3.47\end{array}$ & $\begin{array}{c}<0.001 \\
0.001\end{array}$ & $\begin{array}{c}-0.03(-0.04,-0.02) \\
0.02(0.00,0.03)\end{array}$ & $\begin{array}{c}-4.43 \\
3.78\end{array}$ & $\begin{array}{l}<0.001 \\
<0.001\end{array}$ \\
\hline $\begin{array}{c}\text { Gingiva score } \\
\text { IPA } \\
\text { IPW }\end{array}$ & $\begin{array}{c}-0.02(-0.03,-0.00) \\
0.02(0.01,0.03)\end{array}$ & $\begin{array}{c}0.01(-2.65) \\
0.02-3.77\end{array}$ & $\begin{array}{c}0.008 \\
<0.001\end{array}$ & $\begin{array}{c}-0.02(-0.03,-0.01) \\
0.02(0.01,0.03)\end{array}$ & $\begin{array}{l}-2.91 \\
3.95\end{array}$ & $\begin{array}{c}0.004 \\
<0.001\end{array}$ \\
\hline $\begin{array}{c}\text { Clinical probing depth } \\
\text { IPA } \\
\text { IPW }\end{array}$ & $\begin{array}{c}0.02(-0.02,0.06) \\
0.05(0.02,0.07)\end{array}$ & $\begin{array}{c}0(1.18) \\
0.02(3.45)\end{array}$ & $\begin{array}{l}0.239 \\
0.001\end{array}$ & $\begin{array}{c}- \\
0.05(0.02,0.07)\end{array}$ & $\begin{array}{l}- \\
3.45\end{array}$ & $\begin{array}{c}- \\
0.001\end{array}$ \\
\hline $\begin{array}{l}\text { Bone level } \\
\text { IPA } \\
\text { IPW }\end{array}$ & $\begin{array}{c}0.42(0.01,0.83) \\
-0.55(-0.84,-0.27)\end{array}$ & $\begin{array}{l}0.01(2.01) \\
0.03(-3.83)\end{array}$ & $\begin{array}{c}0.045 \\
<0.001\end{array}$ & $\begin{array}{c}0.54(0.13,0.94) \\
-0.52(-0.80,-0.23)\end{array}$ & $\begin{array}{l}2.59 \\
-3.52\end{array}$ & $\begin{aligned} & 0.01 \\
< & 0.001\end{aligned}$ \\
\hline
\end{tabular}

Adjusted $\mathrm{R}^{2}=0.08 ; \mathrm{n}=661 ; \mathrm{SLR}=$ simple linear regression; and MLR = multiple linear regression; IPA = interproximal contact area; IPW = interproximal width. 


\section{Discussion}

The interproximal space is a pyramidal shape dimension bordered by the interproximal contact area at the peak, interproximal tooth surfaces on the mesial and distal sides, and alveolar bone crest at the base [16]. In the ideal condition, this space should be filled entirely by interdental papilla. Clinically, a precise measurement of the interproximal space is very difficult to achieve if not impossible. Researchers used various measurement methods in an attempt to obtain a close estimation of the interproximal dimension. For example, instead of measuring the surface contact area, the measurement of the interproximal contact area can be represented by the length of contact in an apicoincisal direction [14]. Depending on the objective of the study, the height of the interproximal space can be measured from the interproximal contact area to either; the alveolar bone crest using transgingival probing, radiograph methods or flap reflection methods [17-19]; or the gingival zenith line connecting adjacent teeth [15, 20 21]. Since our study was comparing the healthy and periodontally affected tooth, the gingival zenith line method is thought to be more suitable because of a stable reference point and non-invasiveness. However, due to technical error in the measurement process, data on the interproximal height were excluded from this manuscript. The horizontal distance of the space is even harder to measure since there was no actual marking ever documented for interproximal space. Some studies used the gap between two adjacent roots for the estimation of the horizontal distance of interproximal space [19, 22, 23]. In this study, the distance between gingival zenith points was used to measure the horizontal distance of the interproximal area [24].

The interproximal space is not always fully occupied by the interdental papilla. Observation by Tarnow et al. showed that when the papilla reaches a certain height, the reduced amount of house papilla started to be noticed [18], which usually presented with a round or flat papillary tip, a black triangle. Apart from the obvious aesthetic problem, the black triangle creates an unprotected interproximal area [25], which leads to food impaction that reaches from the facial or lingual/palatal direction. In addition, the reduced fill interdental papilla area complicates the patient oral hygiene care, hence encouraging dental biofilm retention. This study explores how the interproximal space contributes to the biofilm retention and establishment of periodontal disease.

We found in this study that the length of the interproximal contact area has a strong negative relationship with the plaque and gingiva score. The relation was found very weak with periodontal pocket, and no relation was found with bone level. We were unable to locate any similar study that evaluates the relationship between the interproximal contact area and periodontal parameters. However, the presence of the contact point was previously reported in old studies to associate with lesser dental biofilm retention and periodontal tissue inflammation [26, 27]. The size, position and shape of the interproximal contact areas are varied, depending on the factors like location and shape of the contact and tooth contour [28]. A good interproximal contact will stabilise the tooth in the alignment and prevents the contact from separation during function. Thus, acting as an interproximal barrier against food impaction. The small interproximal contact area was also found to associate with increased height of the interproximal area and reduced fill of interdental papilla [29, 30], where the reduced fill of interdental papilla is believed to associate with food impaction and possibly biofilm retention.

In our study, the interproximal width was found to has a strong positive relation with plaque score, gingival score and probing depth, but a negative relation with the bone level. Through our thorough literature search, we have not found any study that specifically looked at the relationship between interproximal width and the periodontal parameters. However, our finding could be explained by the presence of a known direct relationship between the interproximal contact area and width. Increased in the interproximal width means an increase in the distance between teeth, which is most probably associated with a loss or weak in the interproximal contact and reduced the interdental papilla fill. These conditions eventually drive to the dental biofilm formation and retention, and periodontal inflammation [26, 31]. This inferential statement is supported by our multiple regression analyses that showed the interproximal contact area and width are the significant predictors for the models of plaque score, gingiva score and bone level (Table 2).

Interestingly, our data also showed that interproximal width was negatively associated with bone level, contradicting with the finding on clinical probing depth. This is probably best explained by the understanding of the root proximity rule. A human histologic study showed that the quality and quantity of the interproximal bone are determined partially by the distance between the neighbouring teeth, interproximal distance [32]. The close proximity of the bone will reduce the thickness of lamina dura and cause to the meagreness in cancellous bone, which was proposed to create the vulnerability of interdental bone to external forces and inflammation [33, 34]. Lack of cancellous bone quality was also found to associate with reduced bone repair capability and bone loss $[22,33]$.

This study evaluated the relationship between periodontal parameters and the interproximal dimension in the subjects known susceptible to periodontal disease. This intrasubject evaluation approach is advantageous as we can reduce the effect of subject heterogeneity factors such as genetics, age, diet, smoking, alcohol intake or individual oral hygiene practices to the pathogenesis of the periodontal disease $[8,35,36]$. On the other hand, the study used a purposive sampling method, which is subjective and non-probability in nature. This sampling method sets limitations to the study as it creates the possibility of the researcher's bias in making sample selection. Also, in the periodontal parameter measurements, this study used the dichotomous, ordinal scale for measuring plaque and bleeding on probing, which was derogatory. This scale only allows us to determine the presence or absence of plaque and bleeding on probing, and it does not give a true quantitative measure of those variables.

\section{Conclusion}

Within the limitation of the study, we can conclude that the interproximal contact area and width have a significant relationship with the presence of dental biofilm, gingival bleeding and bone level in the subjects susceptible to periodontal disease. The understanding of the role of interproximal dimension in periodontal inflammation may help the clinician in predicting the susceptible teeth for periodontal disease and providing a personalised oral 
hygiene instruction as well as treatment planning. This data gives a preceding for the future studies looking at the role of local factors such as tooth rotation, crowding, drifting and displacement in the susceptibility of periodontal disease.

\section{Acknowledgement}

The study was supported by the Universiti Sains Malaysia (USM) through the USM research grant, Grant no: 304/PPSG/61313137.

\section{References}

[1]. Kumar PS, Leys EJ, Bryk JM, Francisco J Martinez, Melvin L Moeschberger, Ann L Griffen. Changes in periodontal health status are associated with bacterial community shifts as assessed by quantitative $16 \mathrm{~S}$ cloning and sequencing. J Clin Microbiol. 2006; 44:3665-3673. PMID: 17021095.

[2]. Papapanou PN, Wennstrom JL, Grondahl K. Periodontal status in relation to age and tooth type. A cross-sectional radiographic study. J Clin Periodontol. 1988; 15:469-478. PMID: 3263400.

[3]. Shi B, Chang M, Martin J, Makedonka Mitreva, Renate Lux, Perry Klokkevold, et al. Dynamic changes in the subgingival microbiome and their potential for diagnosis and prognosis of periodontitis. 2015; 6(1):e0192614. PMID: 25691586.

[4]. Kornman KS, Loe H. The role of local factors in the etiology of periodontal diseases. Periodontol 2000. 1993; 2:83-97. PMID: 9673183.

[5]. Albandar JM. Global risk factors and risk indicators for periodontal diseases. Periodontol 2000. 2002; 29:177-206. PMID: 12102708

[6]. Lindhe J, Okamoto H, Yoneyama T, A Haffajee, S S Socransky. Periodontal loser sites in untreated adult subjects. J Clin Periodontol. 1989; 16: 671678. PMID: 2613936.

[7]. Gonzalez MK, Almeida AL, Greghi SL, Luiz Fernando Pegoraro, Jose Mondelli, Tatiana Moreno. Interdental papillary house: a new concept and guide for clinicians. Int J Periodontics Restor Dent. 2011; 31:e87-93. PMID: 22140673.

[8]. Taba Jr. M, Souza SL, Mariguela VC. Periodontal disease: a genetic perspective. Braz Oral Res. 2012; 26 Suppl 1:32-38. PMID: 23318742.

[9]. Silva-Boghossian CM, Colombo AP, Tanaka M, Carolina Rayo, Yizhi Xiao, Walter L Siqueira. Quantitative proteomic analysis of gingival crevicular fluid in different periodontal conditions. PLoS One. 2013; 8:e75898. PMID: 24098404

[10]. Tonetti MS, Jepsen S, Jin L, Otomo-Corgel J. Impact of the global burden of periodontal diseases on health, nutrition and wellbeing of mankind: A call for global action. J Clin Periodontol. 2017; 44: 456-462. PMID: 28419559.

[11]. O'Leary TJ, Drake RB, Naylor JE. The plaque control record. J Periodontol. 1972; 43:38. PMID: 4500182.

[12]. Ainamo J, Bay I. Problems and proposals for recording gingivitis and plaque. Int Dent J. 1975; 25:229-235. PMID: 1058834

[13]. Vandana KL, Haneet RK. Cementoenamel junction: An insight. J Indian Soc Periodontol. 2014; 18:549-554. PMID: 25425813.

[14]. Stappert CF, Tarnow DP, Tan JH, Chu SJ. Proximal contact areas of the maxillary anterior dentition. Int J Periodontics Restor Dent. 2010; 30:471-477. PMID: 20814600

[15]. Olsson M, LJndhe J, Marinello CP. On the relationship between crown form and clinical features of the gingiva in adolescents. J Clin Periodontol. 1993; 20:570-577. PMID: 7691897.

[16]. Takei HH. The interdental space. Dent Clin North Am. 1980; 24:169-176. PMID: 6928830.

[17]. De Rouck T, Eghbali R, Collys K, Hugo De Bruyn, Jan Cosyn. The gingival biotype revisited: transparency of the periodontal probe through the gingival margin as a method to discriminate thin from thick gingiva. J Clin Periodontol. 2009; 36:428-433. PMID: 19419444.

[18]. Tarnow DP, Magner AW, Fletcher P. The effect of the distance from the contact point to the crest of bone on the presence or absence of the interproximal dental papilla. J Periodontol. 1992; 63:995-996. PMID: 1474471.

[19]. Cho HS, Jang HS, Kim DK, Joo-Cheol Park, Heung-Joong Kim, Seong-Ho Choi, et al. The effects of interproximal distance between roots on the existence of interdental papillae according to the distance from the contact point to the alveolar crest. J Periodontol. 2006; 77:1651-1657. PMID: 17032106.

[20]. de Santana RB, de Miranda JLC, de Santana CMM. The relationship between open versus normal contact point and inter-proximal papilla dimensions in periodontally healthy young adults: A controlled clinical trial. J Clin Periodontol. 2017; 44:1164-1171. PMID: 28800146.

[21]. Spear FM. Maintenance of the interdental papilla following anterior tooth removal. Pr Periodontics Aesthet Dent. 1999; 11: 21-8. PMID: 10218048.

[22]. Tal H. Relationship between the interproximal distance of roots and the prevalence of intrabony pockets. J Periodontol. 1984; 55: 604-607. PMID: 6593454.

[23]. Trossello VK, Gianelly AA. Orthodontic treatment and periodontal status. J Periodontol. 1979; 50: 665-671. PMID: 294480.

[24]. Yin XJ, Wei BY, Ke XP, Zhang T, Jiang MY, Luo XY, et al. Correlation between clinical parameters of crown and gingival morphology of anterior teeth and periodontal biotypes. BMC Oral Health. 2020; 20:59.

[25]. Nozawa T, Yamaguchi S, Ookame Y, Koichi Shimada, Koji Tanaka, Koichi Ito. The distances between the facial and palatal papillae in the maxillary anterior dentition. Eur J Esthet Dent. 2011; 6: 88-93. PMID: 21403929.

[26]. Hancock EB, Mayo C V, Schwab RR, Wirthlin MR. Influence of interdental contacts on periodontal status. J Periodontol. 1980; 51:445-449. PMID: 6931204 .

[27]. Koral SM, Howell TH, Jeffcoat MK. Alveolar bone loss due to open interproximal contacts in periodontal disease. J Periodontol. 1981; 52:447-450. PMID: 6943330.

[28]. Dörfer CE, von Bethlenfalvy ER, Staehle HJ, Pioch T. Factors influencing proximal dental contact strengths. Eur J Oral Sci. 2000; 108:368-377. PMID: 11037752.

[29]. Chen MC, Liao YF, Chan CP, Yen-Chen Ku, Whei-Lin Pan, Yu-Kang Tu. Factors influencing the presence of interproximal dental papillae between maxillary anterior teeth. J Periodontol. 2010; 81:318-324. PMID: 20151812.

[30]. Sharma AA, Park JH. Esthetic considerations in interdental papilla: remediation and regeneration. J Esthet Restor Dent. 2010; 22:18-28. PMID: 20136942.

[31]. Jernberg GR, Bakdash MB, Keenan KM. Relationship between proximal tooth open contacts and periodontal disease. J Periodontol. 1983; 54:529533. PMID: 6579279.

[32]. Heins PJ, Wieder SM. A histologic study of the width and nature of interradicular spaces in human adult pre-molars and molars. J Dent Res. 1986; 65:948-951. PMID: 3458748 .

[33]. Nielsen IM, Glavind L, Karring T. Interproximal periodontal intrabony defects. Prevalence, localization and etiological factors. J Clin Periodontol. 1980; 7:187-198. PMID: 6933161.

[34]. Kim T, Miyamoto T, Nunn ME, Raul I Garcia, Thomas Dietrich. Root proximity as a risk factor for progression of alveolar bone loss: the Veterans Affairs Dental Longitudinal Study. J Periodontol. 2008; 79: 654-659. PMID: 18380558.

[35]. Marsh PD. The significance of maintaining the stability of the natural microflora of the mouth. Br Dent J. 1991; 171:174-177. PMID: 1910971.

[36]. Kinane DF, Preshaw PM, Loos BG. Host-response: understanding the cellular and molecular mechanisms of host-microbial interactions - consensus of the Seventh European Workshop on Periodontology. J Clin Periodontol . 2011; 38 Suppl 1:44-48. PMID: 21323703. 